\title{
Requirements for Future Photometric and Spectroscopic Observations for Asteroseismology: One Theorist's Perspective
}

\author{
Steven D. Kawaler \\ Iowa State University, Department of Physics and Astronomy, Ames, \\ IA USA 50011
}

\begin{abstract}
The interplay between theory and observation has been particularly close in asteroseismology, ranging from white dwarfs through roAp stars. This trend should continue as the field expands to include new classes such as solar-type stars. This synergistic relationship relies on communication between theorists and observers, but also on healthy skepticism in both directions. Here, I outline a (somewhat personal) view of the theoretical side - what kinds of observations are most helpful for optimizing utility of theoretical research efforts? Where might theory be of closer guidance as observing programs are planned?
\end{abstract}

A few years ago, in a setting long forgotten but during one of the timeless arguments about the relative roles of theory and observation in astronomy, my colleague Darragh O'Donoghue brought up a metaphorical tale of cows and fences. He claimed that theorists are like cows - they graze contentedly, slowly wandering through the pasture in search of fresh grass. Fences surrounding the pasture keep them confined to the farm. Should a hole in the fence exist, the cows would surely wander through the hole and into the wilderness. He likened theorists to the cows, and observers to the farmers who must maintain the integrity of the fences (a metaphor for observational data) lest the theorist-cows wander into the wilderness (of irrelevant theoretical models and ideas).

Though appreciated by this theorist, my argument was that the metaphor works perfectly well with the observers as cows and the theorists maintaining the fences (of interesting objects that can constrain theory) lest the observer-cows wander into the wilderness (of observations of uninteresting objects of limited use in advancing our knowledge of the stars).

We are all cows, and we need our fences. Here, I outline some of the needs that theorists have with regard to observations of pulsating stars for asteroseismological purposes.

What are some useful things that pulsations can teach us about stellar interiors? Working together, theorists and observers can use asteroseismological observations to place tighter constraints on individual global stellar parameters, such as $R, L$, and $M$. Through asymptotic nonradial pulsations, we can learn $\nu_{0}$ and the rotation rate $\Omega$.

With some more work, we can determine the internal composition of stars. This has been amply demonstrated with white dwarf stars (see, for example, Metcalfe, these proceedings, and references therein). Mode trapping effects can 
allow us to find compositional stratification regions (i.e. Kawaler \& Bradley, 1994 and Moskalik et al., these proceedings). This may also be possible for the sdBV stars (see Charpinet et al., these proceedings). Subsurface magnetic fields make their mark on the pulsations of roAp stars, as shown elegantly by M. Cunha (these proceedings). Still more observing work can reveal pulsation period changes caused by secular evolution (i.e. Kepler et al. 2000) that directly confront stellar models and interior physics (i.e. O'Brien \& Kawaler, 2000).

What Theorists Love The job of a theorist is a joyous one when observers do a few relatively simple things. With tight constraints on stellar parameters (via spectroscopy and occasionally, astrometry) we have a reduced phase space to cover with grids of models. Good parameters also provide independent checks of seismic inferences. As one example, see Reed et al. (2000) for an important verification of seismic results for the GW Vir star PG 2131. Accurate determination of stellar parameters (in particular $T_{\text {eff }}$ and $\log g$ ) are important, also, in determining the precise boundaries of instability strips and the distribution of properties across such a strip. If individual stars are moving around in the color-magnitude diagram with time because of revisions of their $T_{\text {eff }}$, that makes pinning down the driving mechanism an even more daunting task.

Theorists also appreciate stars that are "well-behaved", showing pulsation behavior that is expected from simple asteroseismology theory. In those cases, we can fully exploit our tools to to determine stellar interior parameters.

As useful and important as helioseismology has been for a wide variety of fields, it is still just a "point (or line) on a plane" meaning that to explore stellar interiors, the Sun must not be the only subject. We love having several different members of a class of pulsator to fully map out the phase space of stellar physics. With the recent revelations of solar-type oscillations on other stars (Bouchy, and others, these proceedings), life begins anew.

Though we learn much from well-behaved and well-characterized stars, we can learn a great deal from stars with poor observational constraints on their parameters. In these cases, theoretical models can help provide those constraints through asteroseismology. But, beware - underconstrained theory means that the cows may be escaping. Another area where theorists thrive is when considering stars that misbehave - that is, stars with trustworthy data that makes no sense at all. This is a very freeing case that provides fertile ground for imaginative theory that must, eventually, face further observational tests.

Theorists hate chasing "bad data" One of my mentors (Don Winget) is fond of saying that "the only thing worse than no data is bad data" and my experience has borne this out again and again. In the case of asteroseismology, "bad data" usually means mis-identified aliases and misrepresented noise in Fourier transforms.

What we all need are data that are clear. By that, I mean data that are accurately reduced to remove acquisition peculiarities, but are otherwise lightly processed. Surprisingly, observers who use the utmost care in obtaining and reducing photometric time-series data then use antiquated and improper tools in their interpretation and analysis. Power spectra are "CLEAN" ed in efforts to get around sampling aliases, and "false-alarm probabilities" are used in attempts 
to quantify the "reality" of peaks. Experience shows that there is no reliable way to get around the fact that periodic gaps in data mask true periodicities in stars. The underlying noise distribution in time-series photometry from the ground is far from Poisson; our atmosphere and our instruments easily conspire to fool us about what peaks in a spectrum are because a star is varying and which are, in fact, not related to the star at all.

Another place that observers could provide better guidance for theory is that of null results. Most observers have scoured the sky for members of various classes of pulsating star with more null results than successes. The null results remain hidden in private observing logs. Many of us would really like to know about these non-pulsators. Theorists need this information as they try to understand the physics that causes (or in most cases inhibits) pulsations. Observers need to know which stars have already been tested so that they don't waste their time looking at stars that have hours of constant photometry already taken.

After discovery, observers often move on to other targets. But it is followup observations of interesting targets that theorists crave for guidance - we need fully resolved FTs to provide frequencies (and mode identifications) for comparison with models. So many stars look promising based on the discovery papers, but are followed by nothing more in the literature.

What are some new places for observation to help? Theory can provide invaluable in interpreting a variety of data sets. Already mentioned is long-term photometric monitoring. These observations are inexpensive, as they can be done from a single site. They are in general easy to do, and make excellent research projects for undergraduates and beginning graduate students. And, most importantly, they can provide some very sexy results. Long-term monitoring and $(O-C)$ analysis, can detect stellar aging (through $\mathrm{d} P / \mathrm{d} t$ ), stellar "weather" from magnetic activity, and place important constraints on possible planetary and sub-stellar companions.

Large scale photometric monitoring - such as recent microlensing surveys, and ongoing projects including the Sloan Digital Sky Survey, have uncovered a large number of pulsating (and potentially variable) targets such as Cepheids, white dwarfs, $\delta$ Scuti stars, sdBVs, etc. With these large numbers, we can explore "ensemble seismology" with the lack of individual rich pulsators being replaced by statistically significant samples of many stars (Clemens, 1993; Kleinman et al., 2000; Handler et al., these proceedings). This sort of analysis can reveal class properties and thereby tightly constrain stellar evolution.

Another new and exciting area involves pulsating stars in clusters. While pulsating DA white dwarfs in globular clusters will not be explored through timeseries photometry soon, the DBV and GW Vir stars, were they to be found in globular or open clusters, should be bright enough for seismic observations with large telescopes. Even more promising is the chance of finding pulsating $\mathrm{sdB}$ stars in open and globular clusters.

Finally, Simon O'Toole (these proceedings, and references therein) reports successful time--series measurement of radial velocity pulsations in an sdBV star (PG 1605). Observations such as these hold promise for providing mode identifications for these stars and, more intriguingly, in sampling the pulsation eigenfunction near the surface of these stars. Such observations will directly confront 


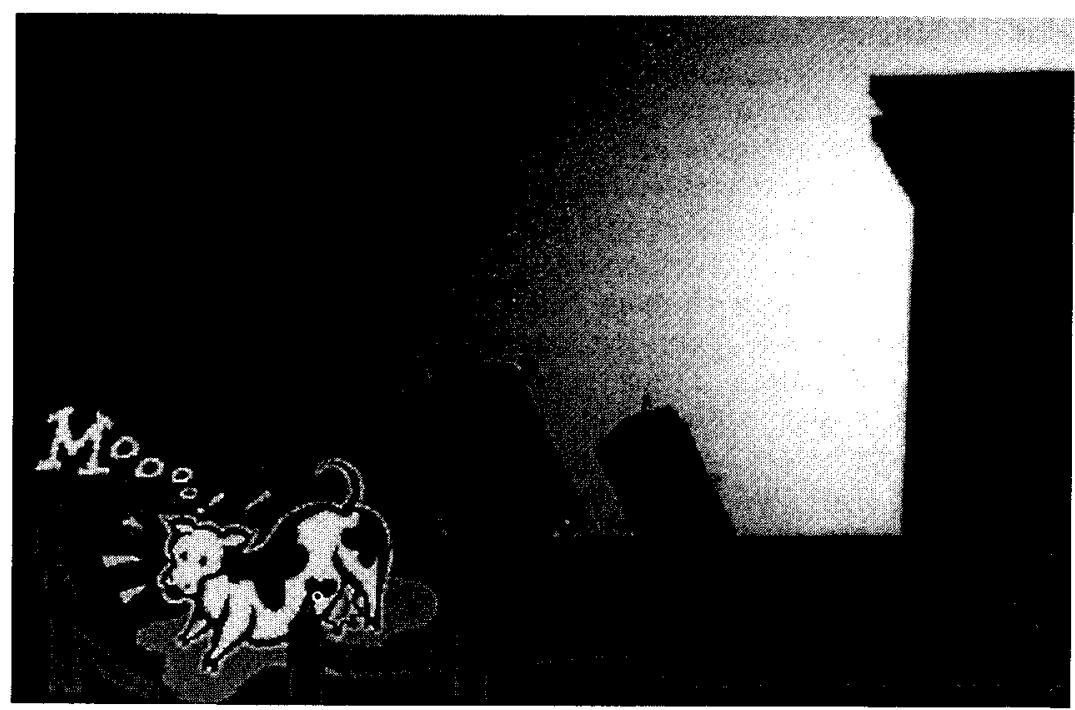

Figure 1. Data fences need to be maintained lest theorists wander through the holes into the wilderness. Observers too need to mind the limits of theory lest they move away from observing targets of use and interest. After O'Donoghue (1995).

the theoretical models, in ways similar to the work by Baldry \& Bedding (2000) on roAp stars.

Conclusions So, in conclusion, what are the needs of theorists for future seismic observations? First of all, as Luis Balona has demonstrated, mode identification is crucial - without knowing the values of $\ell$ and $m$ at least, models will be underconstrained. Second, null results are very important! Without knowing if a star has been tested for variability, or if short (or long) period modes are present in a known pulsator, we may pursue the theoretical possibilities at length with no observations to explain. Third, discovery of new pulsators, while exciting and interesting, is rarely valuable after the $n$th member. Follow-up observations of interesting targets, which takes time, can provide the needed data to resolve fully the pulsations, and track any long-term changes. That is the sort of information that yields potentially breakthrough discoveries about the stars. Fourth, it would be very valuable to have accessible data archives of timeseries photometry and spectroscopy, both in raw and reduced form. Published plots and tables only go so far - some important effects that the models address are not testable with FTs and period lists that are the common final product of a paper, but need full time-series information.

In closing, I (as one theorist) loudly applaud the terrific work of my observing colleagues, and will continue to cheer loudly from the sidelines. Keep a close 
eye on your window patterns, though. And trust theory as a guide, and only a guide, to your future programs. We all are cows, and we need our fences.

\section{References}

Baldry, I. \& Bedding, T. 2000, MNRAS, 318, 341

Clemens, J.C. 1993, PhD Thesis, University of Texas, Austin

Kawaler, S. \& Bradley, P. 1994, ApJ, 427, 415

Kepler, S.O., et al. 2000, ApJ, 534, L185

Kleinman, S.J., Kawaler, S.D., \& Bischoff, A. 2000, in ASP Conf. Ser., Vol. 203, The Impact of Large-Scale Surveys on Pulsating Star Research, eds. L. Szabados \& D. Kurtz (San Francisco: ASP), 515

O'Brien, M.S. \& Kawaler, S.D. 2000, ApJ, 539, 372

O'Donoghue, D.O. 1995, private communication

Reed, M.D., Kawaler, S.D., \& O'Brien, M.S. 2000, ApJ, 545, 429.

\section{Discussion}

A. Cox : What do the latest results tell us about the hydrogen layer thickness in the ZZ Ceti stars?

S. Kawaler : Based on the Kleinman et al. (2000) analysis of the data as of Clemens (1993), it looks like we have a good handle on both the hydrogen layer thickness (at the "evolutionary" value of $10^{-4} M_{*}$ ) and, more firmly, the helium layer thickness of $10^{-2} M_{*}$. 